\title{
Quantifying Crystallographic Information from Electron Diffraction Patterns in the TEM
}

\author{
JL Lábár \\ Thin Film Physics Department, Research Institute for Technical Physics and Materials Science \\ (MTA MFA), H-1121 Budapest, Konkoly-Thege M. u. 29-33, Hungary \\ In the last chapter of his marvelous Thesis, Castaing described how to extract crystallographic \\ information from micron sized crystallites under the electron beam of a microprobe [1]. Even \\ though he used the x-rays, the so called the Kossel-lines, generated locally by the electron beam \\ of the microprobe, similar information can also be obtained from the pattern formed by the \\ diffracted electrons both in an SEM (EBSD) and in a TEM. Present paper summarizes the types \\ of information obtainable by the "ProcessDiffraction" method from different types of electron \\ diffraction patterns in the TEM. Citation of other similar methods can also be found in the \\ references below.
}

First, characterization of individual single crystallites is given. Second, quantitative processing of patterns from a large assembly of nanocrystals is presented. Third, deduction of the short range order in amorphous films is described.

A single crystal in a TEM practically starts with linear dimensions of the order of $10 \mathrm{~nm}$ (in any directions) up to about $200 \mathrm{~nm}$ in beam direction and unlimited size in lateral directions. Two typical tasks to solve with spot patterns from almost parallel illumination (SAED or microdiffraction) are deciding if an assumed crystalline phase can produce the measured pattern and determining the approximate orientation of the crystallite (zone axis of the pattern). One problem is posed by the limited accuracy of the experimental data, due to both the loose practice followed by many microscopists and the length of the relrods (in thin samples). The remedy for the first problem is a standardized experimental protocol, suggested in [2]. Ambiguity of phase identification is removed by the simultaneous processing and solving of an entire set of (in contrast to only one) diffraction patterns, recorded in a tilt series, with known goniometer positions [3]. Although, there might be chance that all the measured parameters (distances and angles) from a single experimental pattern are within the experimental error to the corresponding values from two (or more) different calculated patterns, this chance quickly converges to zero when more and more (differently oriented) sections of a 3D reciprocal lattice are recorded and processed together as members of the same set.

If convergent beam electron diffraction (CBED) patterns are recorded from thicker $(>50 \mathrm{~nm})$ single crystals, they frequently contain Kikuchi-lines and Kikuchi-bands. These patterns are generally recorded with low camera length to include the maximal possible angular range in the experimental pattern. Identification of 3 different Kikuchi-bands facilitates accurate determination of the local orientation of the measured crystallite. Exact orientation is important when relative orientation of two neighboring grains is to be determined, while geometrical characterization of a grain boundary is aimed at. 24 , symmetry related orientation matrices $\left(\underline{\mathrm{O}}_{\mathrm{i}}\right)$ are calculated for a single grain in a cubic system to specify the orientation of the crystal coordinate system as compared to the orientation of the coordinate system of the recorded CBED pattern. Both the axis and the angle of rotation (between two neighboring grains) are calculated from the diagonal elements of the misorientation matrix $\mathrm{M}=\underline{\mathrm{O}}_{1}{ }^{*} \underline{\mathrm{O}}_{2}{ }^{-1}$. Experimental determination 
of the grain boundary (GB) plane, between the two grains, needs additional measurement of the trace of the GB-plane in a BF image and calibration of the rotation of the TEM.

Nanocrystalline thin films, producing ring patterns, are frequently encountered in TEM laboratories. Determination of the fractions of the different crystalline phases and their possible texture is of importance for most of them. A method, similar to the Rietveld-method, was developed for this purpose within ProcessDiffraction [4], [5]. Transformation of the 2D measured SAED pattern into a 1D distribution produces peaks over background, similarly to the everyday X-ray powder diffraction. Diffraction lines of known phases are calculated for electrons using the known atomic positions. Correction for small dynamic effects is included by using the Blackman approximation with the Bethe effective potential incorporated. Adding peak-shapes to the lines, the measured diffraction peaks are modeled, together with an empirical modeling of the background. Parameters of the model include peak-shapes, experimental parameters (like camera length fine-tuning) and sample parameters (like volume fraction and textured fractions of phases). Best solution is obtained by minimizing the deviation between modeled and measured distributions by a non-linear least square fitting procedure, while probing the parameter space with the SIMPLEX method. Fiber texture can only be included in the model for the present implementation of the method. First fitting, attempted with models, calculated for random orientation distribution gives hint for the possible texture (by showing which diffraction lines are under represented and which ones are over represented in the measured distribution). Good examples with both cubic and hexagonal phases were obtained.

Short range order can be examined in thin films from the broad, diffuse rings recorded from amorphous (or nanocrystalline) samples. Kinematic diffraction condition is an important requirement for the experimentally recorded patterns, putting a strong constrain on the useful samples. Fourier-transform of an appropriately normalized diffraction pattern (the reduced interference-function) gives the pair distribution function, which is the envelope of the partial pair-distribution functions. Although the partials can not be directly resolved with electron diffraction alone, the dominant atomic distances can frequently be determined from the envelope, provided the number of components is limited in the thin film [2], [6]. Empirical correction of experimental problems on the basis of physical constraints is an important part of the iterative procedure.

The method, implemented in a computer program that runs on IBM PC machines under the Windows operating system can be downloaded free from the home page of the author [7]

References

[1] R. Castaing, Thesis (1951) Univ. Paris

[2] V. Kovács Kis, M. Pósfai, J.L. Lábár, Atmospheric Environment 40 (2006) 5533-5542.

[3] J.L. Lábár, Ultramicroscopy 103 (2005) 237-249.

[4] J.L. Lábár., Microsc. Microanal. 14 (2008), 287-295.

[5] J.L. Lábár., Microsc. Microanal. 15 (1) (2009) 20-29.

[6] J.L. Lábár, A. Kovács, B.P. Barna, T. Hanada, M. Ishimaru, Y. Hirotsu, I.T. Bae, Proc. 6th Multinational Congress on Electron Microscopy, Pula (2003), 469-470.

[7] http://www.mfa.kfki.hu/ labar/ProcDif.htm 